\title{
LA PENSÉE ICONIQUE
}

\author{
Baptiste Campion et Philippe Verhaegen
}

L'approche des phénomènes de communication a fait l'objet de nombreux points de vue, chacun d'eux s'évertuant à mettre en exergue telle ou telle caractéristique du phénomène. Ainsi, pour faire court, on peut considérer qu'il existe trois grandes "tendances" historiques dans les sciences de l'information et de la communication. Des liens étroits existent bien entendu entre ces tendances, que nous distinguons toutefois pour la clarté du propos. La première, essentiellement sémiotique et basée sur la linguistique saussurienne, se centre sur les structures et les conventions qui sous-tendent l'articulation des "signes" au sein du message, ainsi que sur les rapports entre celui-ci et le référent. La seconde orientation s'attache aux dynamiques interactionnelles. On y retrouvera notamment certaines approches anthropologiques, mais également les travaux en pragmatique linguistique qui étudient les interactions et la manière dont les individus en interaction contribuent à la co-construction du sens et se forgent des règles d'interaction et référentiels communs. Enfin, la troisième orientation, historiquement plus récente, envisage l'articulation entre les processus de sémiotisation inhérents aux messages et la construction intra-psychique de la communication, c'est-à-dire les processus cognitifs. C'est dans ce dernier cadre que l'interrogation sur la nature de la pensée, issue plutôt des sciences cognitives et de la philosophie de l'esprit, apporte des éléments importants à la recherche en communication. Décrire la manière dont se construisent les représentations mentales permet de comprendre la manière dont l'individu va construire le sens.

Recherches en communication, $\mathrm{n}^{\circ} 29$ (2008). 
Le champ des sciences cognitives est investi par des approches multiples nourries par des travaux issus de disciplines aussi diverses que la psychologie, la philosophie, la linguistique, les neurosciences, ... Très (trop) rapidement, on peut dire que la recherche dans ce domaine s'est soit centrée sur une approche objectiviste de la cognition - l'esprit humain est alors considéré comme un système de traitement de symboles- soit centrée sur une approche plus subjective, l'expérientialisme, qui accorde au corps et à l'environnement social et culturel un rôle déterminant dans le développement et le fonctionnement de la cognition ${ }^{1}$. C'est dans cette seconde voie que s'est véritablement développée une réflexion sur le rôle et la place de l'image au sein des processus cognitifs.

Les sciences cognitives ont ainsi mis en avant des notions comme celles de représentation et d'image mentale ${ }^{2}$. La théorie des modèles mentaux $^{3}$ a pu servir de base à des travaux montrant la nature "imagée" (iconique) de ces représentations, par opposition aux théories propositionnalistes qui envisagent les processus cognitifs comme manipulant essentiellement des symboles. Et même plus : "bien souvent le langage peut être le déclencheur d'une activité d'imagerie"', comme le soulignent Jean-Pierre Meunier et Daniel Peraya, mettant en avant la dimension spécifiquement iconique des représentations mentales, qui s'appuient sur un langage propositionnel ou sur une perception sensori-motrice de l'environnement ${ }^{5}$.

Pour le chercheur en communication, la prise en compte de ces résultats permet de s'interroger sur la manière dont les messages contribuent à construire, chez le récepteur, une représentation du monde. Basiquement, on dira que le message "efficace" —avec toute la prudence avec laquelle il convient de manipuler ce qualificatif - est un message qui permet la mobilisation chez le récepteur de représentations mentales antérieures

1 J-P. MEUNIER, “Les paradigmes des sciences cognitives", in Approches systémiques de la communication, Bruxelles, De Boeck, 2003, pp. 129-165

2 M. DENIS, Image et cognition, Paris, PUF, 1989.

3 Voir notamment Ph. JOHNSON-LAIRD, "Mental models in cognitive", in Cognitive science, $\mathrm{n}^{\circ} 4,1980$, pp. 71-115. Ces notions ont été largement débattues dans les pages de Recherches en communication : voir en particulier J-P. MEUNIER et A. BERTEN, "A propos de Philip Johnson-Laird, L'ordinateur et l'esprit", in Recherches en communication, ${ }^{\circ} 4,1995$, pp. 243-256.

4 J-P. MEUNIER et D. PERAYA, "Sémiotique et cognition : voyage autour de quelques concepts", in VOIR. L'image mentale, vol.I n¹6, mai 1998, pp. 16-28.

5 J-P. MEUNIER et D. PERAYA, op. cit. ; J-P. MEUnIER, "Connaître par l'image", in Recherches en communication, $\mathrm{n}^{\circ} 10,1998$, pp. 35-75. 
et l'enrichissement de ces structures (ou l'émergence de nouvelles) au départ des éléments donnés dans le message, de sorte que les représentations qui en résultent soient compatibles avec l'intention du destinateur. La dimension strictement iconique de la pensée intervient, dans ce questionnement, dans le mécanisme de construction et d'intégration de ces représentations.

Nous pourrions, dans cette présentation, "définir" la pensée iconique par quelques questions que pose au chercheur cette perspective sur la communication : quel(s) rapport(s) y a-t-il entre les caractéristiques sémiotiques des représentations externes et la forme de la représentation interne ? Dans la conception d'un message, quelle est la prise en compte des représentations internes (et dans quelle mesure) ? Les représentations externes sont-elles un support pour les opérations "internes" de traitement menées par le sujet... et inversement ? Sans prétendre parvenir à faire le tour de ces questions, elles seront abordées, sous différents angles et, généralement, sur base de l'étude d'objets concrets, dans les contributions constituant ce dossier thématique.

D'une certaine manière, celui-ci peut être vu comme un prolongement des travaux présentés il y a dix ans dans une précédente livraison de Recherches en communication ${ }^{1}$ sous l' Intitulé "Image(s) et cognition". Ce numéro faisait suite aux abondants travaux menés jusque-là sur les usages pédagogiques de l'image et des médias dans un contexte où s'étaient multipliés les thématiques et supports de communication (notamment avec le développement des médias électroniques vus comme "technologies de l'intelligence" selon l'expression fameuse de Pierre Lévy). Mais, soulignait alors Daniel Peraya, "l'analyse du rapport entre communication et cognition ne concern[ait] plus le seul champ de la communication éducative où ce rapprochement avait été initialement théorisé"2, mais débordait largement ce cadre pour marquer profondément les modèles théoriques linguistiques et les études portant sur l'image. Ce numéro se voulait programmatique en présentant des contributions théoriques ou des études de cas visant à proposer ou tester des hypothèses relatives à l'articulation des représentations matérielles et des images mentales. Ces pistes de travail ont donné lieu à différents travaux qui ont permis de développer la connaissance des phénomènes de compréhension et construction de sens à l'œuvre chez l'utilisateur de médias.

1 "Image(s) et cognition", Recherches en communication, n¹0, 1998.

2 D. PERAYA, "Image(s) et cognition", présentation du dossier, Recherches en communication, $\mathrm{n}^{\circ} 10,1998, \mathrm{p} .12$. 
Dix ans plus tard, nous proposons de mesurer une partie du chemin parcouru en nous intéressant spécifiquement à la dimension iconique du raisonnement telle qu' elle est mobilisée dans le cadre des (ou suscitée par les) échanges communicationnels, notamment médiatiques. La première phase de travail sur ces questions s'est articulée autour d'un colloque organisé à l'occasion de l'accession à l'éméritat de Jean-Pierre Meunier, professeur au Département de communication de l'UCL pendant 40 ans et initiateur de cette revue (dont il a par ailleurs assuré la direction depuis le premier numéro). Ce colloque se proposait de faire un état des lieux des travaux de recherche récents concernant les approches cognitives de la communication et de la place des images (matérielles et mentales) dans celle-ci. L'objectif était, d'une part, de retracer l'évolution du regard porté par les sciences de la communication sur les objets de recherche auxquels Jean-Pierre Meunier s'est intéressé tout au long de sa carrière, et d'autre part faire le point sur les approches théoriques à partir desquelles il les a abordés ces dernières années. Dans ce cadre, les contributeurs ont abordé, sur des objets spécifiques, cinq grandes questions :

- La nature des représentations mentales;

- La place des représentations mentales et matérielles dans les processus interprétatifs et leur interaction au sein de ceux-ci ;

- L'étude comparée des systèmes de représentation iconiques et des registres sémio-cognitifs qu'ils mobilisent ;

- Les relations entre opérations filmiques et opérations cognitives : c'est-à-dire d'une part l'analyse sémiotique des traitements filmiques et d'autre part celle de l'activité cognitive et des formes de représentations mentales que ces traitements illustrent ou produisent;

- Les implications de ces questions pour le domaine de l'éducation formelle et informelle.

La seconde phase de travail a consisté à approfondir ces questions et à constituer le présent dossier. Il ne s'agit donc pas stricto sensu des actes du colloque qui s'est tenu en avril 2008 même si une bonne part des contributions - retravaillées — ont été reprises ici. Nous l'avons organisé en trois temps.

L'article d'Adriano Duarte Rodrigues forme le premier et se situe, en quelque sorte, en amont de notre interrogation spécifiquement communicationnelle. Son texte propose une réflexion sur le rôle de la mémoire dans la construction des images, proposant une comparaison entre images mentales, verbales et matérielles, ainsi que sur la place que prennent dans ce processus les dispositifs techniques de production et de représentation. La mémoire, nous dit l'auteur, joue un double rôle dans la constitution 
des images : elle assure le découpage de l'expérience en prélevant du flux continu les éléments qui constitueront la représentation, et elle établit le rapport des éléments représentés avec le hors-champ, délimitant par la même occasion les frontières du monde représenté.

Dans un second temps, nous proposons des contributions interrogeant l'articulation entre représentations mentales et matérielles au départ de dispositifs spécifiques de communication. Certains articles utilisent leur objet d'étude comme moyen d'interroger les relations entre images matérielles et la dimension cognitive qu'elles supportent ou alimentent. C'est le cas de Jacques Polet, qui propose une réflexion sur les rapports entre sensorialité et image, sur base d'une analyse d'un film de Chaplin, essayant de montrer comment l'image peut mobiliser les différents sens. En particulier, il montre comment ce film muet peut, sur base de la seule représentation iconique, solliciter un sens autre que celui habituellement mobilisé par l'image auprès d'un sujet en situation spectatorielle dont il est en quelque sorte abstrait pour être plongé dans un univers sensoriel différent. Baptiste Campion et Pierre Fastrez adoptent une démarche semblable d'interrogation sur les rapports entre images mentales et matérielles au départ des caractéristiques propres à un objet particulier : les jeux vidéos. Les auteurs cherchent d'une part à voir dans quelle mesure les cadres conceptuels forgés pour l'analyse de médias audiovisuels plus anciens fournissent une grille de lecture adaptée aux possibilités actuelles du multimédia, et d'autre part à dégager ce qui peut être proposé sur cette base en termes d'effets cognitifs associés à ces dispositifs médiatiques.

Inversement, d'autres contributions s'interrogent sur la dimension spécifiquement iconique de dispositifs communicationnels. Il ne s'agit plus seulement de voir comment un objet d'étude permet de poser la question de l'image, mais également de voir ce que la dimension iconique de la pensée permet de comprendre du fonctionnement d'un dispositif communicationnel particulier. Daniel Peraya et Anne-Sophie Collard utilisent ainsi l'approche cognitive de la métaphore proposée par Lakoff et Johnson, pour à la fois mieux asseoir la définition d'un objet particulier, le campus virtuel, et mieux comprendre le processus métaphorique luimême. Cela leur permet d'avancer la proposition que la nature indicielle de la métaphore susciterait chez les utilisateurs de campus virtuels une expérience spécifique de l'espace, plus proche de l'expérience sensible du campus que de la manipulation de représentations. Philippe Verhaegen va plus loin, en montrant comment une perspective strictement sémiotique peut (doit ?) être dépassée et élargie à la dimension corporelle et 
spatiale au sujet des musées et expositions, seuls médias qui prennent à ce point ces dimensions en compte. De la même manière, dans son article, Anik Meunier cherche à définir plus précisément l'éducation muséale, qui correspond avant tout à une série de pratiques professionnelles, parfois assez disparates. L'objectif de l'auteur est de proposer une définition de l'éducation muséale comme champ disciplinaire autonome, et à cette fin en présente de façon critique les modèles émergents.

Enfin, dans un troisième temps, nous proposons deux contributions de chercheurs ayant beaucoup travaillé la thématique des images et, de ce fait, cherchent à envisager des pistes de dépassement des approches de cet objet. D'abord, l'article de Bernard Darras et Sarah Belkhamsa déplace le questionnement vers les objets physiques qui étaient représentés dans ces images. Les auteurs montrent que la théorie sémiotique pragmatique offre des outils suffisamment généraux pour conduire une enquête sur la culture matérielle en accentuant les dimensions de l'action et de la pratique. Mais pour ce faire, il convient de revoir les fondements épistémologiques de la recherche sémiotique en les confrontant aux théories écologiques et énactives cherchant à comprendre les modalités de l'expérience ainsi que le traitement de l'information dans l'action. Cette interrogation occupera l'essentiel de leur texte.

C'est Jean-Pierre Meunier qui clôturera ce dossier. Il était normal de donner "le mot de la fin" à celui dont les travaux ont guidé non seulement la constitution de ce dossier mais également une part importante des travaux de certains de ses contributeurs. De plus, cet article "boucle la boucle" en retraçant d'une part —à partir de son expérience personnelle- l'historique des études ayant abordé l'image et en proposant d'autre part quelques lignes directrices pour les recherches à mener sur cette thématique. Il prône ainsi pour l'étude de l'image un retour à l'interdisciplinarité qui a pu porter les sciences de la communication, et souligne les apports de la sémantique cognitive qui permet d'approfondir la compréhension des images parce qu'elle provient, dit-il, d'un champ de la linguistique ayant retrouvé la dimension iconique sous-tendant toute communication verbale. 